\title{
A Concise Review on Microspheres for Targeted Drug Delivery
}

\author{
Bissera Pilicheva* \\ Department of Pharmaceutical Sciences, Medical University-Plovdiv, Bulgaria
}

Submission: April 04, 2018; Published: April 16, 2018

*Corresponding author: Bissera Pilicheva, Department of Pharmaceutical Sciences, Medical University-Plovdiv, Bulgaria, Tel: +359-888-651-857; Email: bisserapi@gbg.bg

Keywords: Polyvinyl alcohol; Microparticles; Sodium acrylate polyvinyl; Beclomethasone; oxymetazoline; Quadrasphere®; Hepasphere®; Nanoparticles

\section{Introduction}

In recent years, there has been a growing number of studies demonstrating that microspheres have a strong potential for selective drug delivery and highlighting the ability to target these carriers to different parts of the body using sophisticated delivery technologies. Microspheres are solid, spherical particles of $1-1000 \mu \mathrm{m}$ in size, most often made of a polymer matrix in which the active substance is dispersed at molecular or macroscopic level. The use of mucoadhesive polymers for the construction of the polymer matrix leads to the formation of particles prone to prolonged retention of the applied sitemucoadhesive microspheres Siepmann [1]. Although micro carriers might be used for targeted delivery of drugs in different therapeutic areas, the need for precise selective introduction of drug molecules into targeted tissues and organs is perhaps of greatest significance in the treatment of oncological diseases.

In liver cancer, microspheres are usually used as a bridge to surgical intervention, before transplantation or radiotherapy One of the main routes of cytoreductive therapy involves transvascular drug administration. The numerous approaches that use the trans-arterial pathway are based on the fact that, unlike normal hepatocytes, metastatic tumors are being supplied by the arterial bloodstream rather than by the portal. The introduction of the drug into the liver arteries allows preferential delivery to the peritumoral vascular plexus. For example, injection of a suspension of particles (microspheres) of appropriate diameter into the liver artery results in their accumulation mainly in the peritumor vessels, a process called embolization [2]. Combining a high-energy source of radiation with intra-arterial delivery of embolizing microparticles of appropriate size allows affecting primarily the tumor tissue. Radioisotopes such as yttrium-90
(Y-90) provide an area of high radiation exposure in the tumor and its vicinity, while maintaining normal hepatic parenchyma exposure within the acceptable levels, creating premise for radio immunization [3]. The described approach has found a real application in the SIRT-therapy. SIRT (Selective Internal Radiation Therapy) is an innovative treatment method based on the introduction of radioactive microspheres in liver tumors. Microspheres, usually about $30 \mu \mathrm{m}$ in diameter, are made of glass or polymer resin and contain Y-90 which is bonded either to their surface or to other part of their structure. There are currently two different types of microspheres. Thera-Sphere $₫$ contains millions of glass micro-pearls and SIR-Spheres ${ }^{\circledR}$ consists of polymer beads. Radioactive microspheres emit beta particles whose penetration capacity is around $2.5 \mathrm{~mm}$. Microspheres provide local radiation therapy for liver tumors by simultaneously embolizing blood vessels (blocking small arteries from the liver artery). After injection, the microspheres deliver over $95 \%$ of their radioactive dose within two weeks [4].

Various strategies can be used to selectively target drug release to the colon. Medicines are usually delivered to the colon after coating with polymers such as cellulose derivatives or polyacrylates. However, the effectiveness of such systems may be limited by gastrointestinal motility and variations in $\mathrm{pH}$. Micro carrier systems have been developed to overcome these constraints. Guar gum microspheres are a potential system for targeted delivery of methotrexate in colorectal cancer chemotherapy. The microspheres retain the drug until it reaches the colon, where bacterial enzymes break down the polymer and allow drug release in the targeted area [5].

PLGA microspheres loaded with paclitaxel significantly inhibit the growth of Lewis lung carcinoma cells in vivo without clinically proven toxicity [6]. Mesopore spheres chemically 


\section{Global Journal of Pharmacy \& Pharmaceutical Sciences}

modified with various surface molecules (lipid, amino terminus linker, thiol group or tetraethylene glycol) are effective carriers for targeted delivery of chemotherapeutic drugs to the lungs. By differential count of cells in bronchoalveolar and pleural fluid, these carriers are found to be non-immunogenic and non-toxic [7]. Conjugation of camptothecin to pegylated microspheres prolongs release of the drug in vitro and improves in vivo antitumor efficacy in a rat model of orthotopic lung cancer [8]. Microparticles degradable by the enzyme elastase were prepared by cross linking of alginate in the presence of elastin, an elastase substrate, using Ca2+ ions. The release of protein load in response to a biological signal in the presence of elastase is evidence of enzymatic-specific particle degradation. The microparticles exhibit beneficial properties such as high encapsulation efficiency and good powder dispersibility. Potential polymer toxicity in the lungs was assessed by deposition of the microparticles on Calu-3 cell monolayer and monitoring the changes in transepithelial electrical resistance and induction of cytokine release.

The microparticles show no toxic or immunogenic effects [9]. Chitosan microspheres, cross linked with glutaraldehyde, have been proposed to deliver docetaxel to the lungs with appropriate physicochemical properties and particle size ranges. Microspheres have been found to release the drug to the maximum extent in the targeted tissue [10]. A novel technique has been patented for the preparation of albumin microspheres for salbutamol delivery, which enables the production of particles with high yield and drug loading. Microspheres release the drug for 12 hours in vitro, and in an animal model predominantly accumulate in the lungs than in the blood, the spleen and the liver [11]. Carbopol microspheres have been developed for targeted delivery of methotrexate to the lungs. The study showed an increased concentration of the substance in the lungs compared to that in the blood, spleen and liver, and histopathological assays confirmed the safety and tolerability of the excipients used. This confirms that microspheres are one of the most suitable carriers for drug delivery to the lung [12].

Despite the rapid development of science toward the design of novel drug delivery systems, targeted delivery to the CNS remains challenging. A microparticulate system has been developed to supply 5-fluorouracil to the brain based on poly (methylidene malonate) polymer, which features a low rate of degradation, determining the long-term effect of the drug delivery system [13]. Thanks to the unique connection of the brain to the nasal cavity via the olfactory neurons, delivery of drugs to the CNS can take place within a few minutes if the dosage form is applied to the olfactory area of the nasal cavity. Powder formulations containing mucoadhesive polymers have been investigated back in 1984 by Nagai et al. [14] for nasal administration of peptides and proteins. In the following years, a number of ex vivo and in vivo studies have been published on the influence of mucoadhesive polymers on the mucocilliary transport of various substances such as interferon, beclomethasone, oxymetazoline
[15]. The mucoadhesive properties of albumin microspheres were investigated [16] and prolongation of the residence time in the nasal cavity was established due to the formation of a gellike layer that is slowly eliminated by mucocilliary clearance. Increased absorption of metoclopramide and desmopressin incorporated into microspheres with starch was achieved [17]. Chitosan microspheres have been studied for the delivery of methotrexate to the CNS and show increased absorption of the drug to a nasally administered aqueous solution, demonstrating the ability of the polymer to increase the absorption of poorly absorbed substances [18]. A study on clonazepam distribution in rat CNS after nasal administration of gelatin-chitosan microspheres showed higher brain drug levels than those following intravenous administration of a solution [19].

A relatively new direction in antitumor therapy, which comprises the application of particles, e.g. microspheres capable of releasing drugs is the so-called chemoembolization. This innovative technology focuses on achieving higher drug concentrations in the tumor and lower plasma levels compared to conventional cancer chemotherapy. For this purpose, drug carriers need to possess some essential features such as accurate delivery and controlled/prolonged release, ability to maintain high intra-tumor concentration for a long time without causing damage to surrounding tissues. They may contain non-degradable polymers such as polyvinyl alcohol (PVA) or biodegradable materials such as PLGA [20,21]. Microspheres of sodium acrylate polyvinyl (SAP) have been developed, which have been applied for several years for embolization of hepatocellular carcinoma. They have been approved under the name HepaSphere ${ }^{\circledR}$ (EMA) and QuadraSphere ${ }^{\circledR}$ (FDA) for the treatment of primary and metastatic liver tumors. SAP microspheres are spherical embolization agents derived from a polyvinyl alcohol-sodium acrylate copolymer. They are able to absorb drug molecules via an ion exchange process. SAP microspheres are available as powders in several calibrated plural 50-100, 100-150 and 150-200 $\mu \mathrm{m}$ and can quickly absorb aqueous medium in an amount up to 64 times higher than their original volume, keeping the spherical shape $[22,23]$. Over the last two decades, calibrated microspheres have revolutionized embolization since they ensure a precise and effective execution of the procedure and enable its control by varying the amount of injected material. A moderate embolization approach is the application of a combination of degradable starch microspheres and iodinised oil (Lipiodol) to unite antitumor efficacy and low toxicity in patients stratified for hemoembolisation [24]. It has been found that 1,25-dihydroxy vitamin D3 can inhibit the proliferation of cancer cells, including colorectal and hepatocellular. Microspheres of poly (vinyl neodecanoate) crosslinked with ethylene glycol dimethacrylate are administered as a vehicle for the controlled release of 1,25-dihydroxyvitamin D3 at the target sites for an extended period of time. Cytotoxicity tests reveal the possibility of using this polymeric carrier for targeted drug delivery to the liver, demonstrating high efficacy and optimal bioavailability [25]. 


\section{Global Journal of Pharmacy \& Pharmaceutical Sciences}

Helmuth Möhwald et al [26]. have developed a technique for producing microspheres for targeted drug delivery using porous calcium carbonate microspheres as patterns for the production of hollow structures. Researchers have demonstrated by using inorganic nano-objects as building blocks with polyelectrolyte encapsulation, those three-dimensional hybrid colloidal spheres with integrated functions and collective properties can be produced by a simple pattern strategy. Scientists use microspheres of calcium carbonate and fill their pores with nanoparticles, wrap them with a network of long-chain proteins or polymer filaments, then dissolve the carbonate template in acid. Nanoparticles are arranged in a porous sphere wrapped in the protein network. The protein panel not only envelops the hollow sphere but is also biocompatible and may contain biochemical signaling substances that send the spheres directly to their target in the body.

Microspheres are a unique carrier system for a large number of drugs and can be adapted to adhere to different tissues, cancers located in the respiratory, digestive and urinary system. They can be used not only for controlled release, but also for targeted delivery of drugs to certain parts of the body. Recent advances in medicine predict the development of polymer delivery systems for protein/peptide drugs and in gene therapy. Although significant progress has been made in the field of microspheres, there are still many challenges in this field. The most significant are the development of universally acceptable standard assessment methods and the development of newer polymers with targeting properties. Polymer science must be thoroughly studied to find new value-added polymers in terms of biodegradability, biocompatibility and bioadhesion. A multidisciplinary approach will be needed to overcome these challenges and use microspheres as the most advanced technology for directed and controlled release of new and existing drugs.

\section{References}

1. Siepmann J, Siepmann F (2006) Microparticles used as drug delivery systems. Progr Colloid Polym Sci 133(2): 15-21.

2. Agrawal P, Rajput M (2010) Microspheres in cancer therapy. Ind J Cancer 47(4): 458-468.

3. Murthy R, Habbul A (2006) Trans-arterial hepatic radioembolisation of yttrium-90 microspheres. Biomed Imag Interven J 2(3): e43.

4. Allison C (2007) Yttrium-90 microspheres (TheraSphere and SIRSpheres) for the treatment of unresectable hepatocellular carcinoma. Issues Emerg Health Technol (102): 1-6.

5. Chaurasia M, Chourasia MK, Jain N, et al. (2006) Cross-linked guar gum microspheres: a viable approach for improved delivery of anticancer drugs for the treatment of colorectal cancer. AAPS PharmSciTech 7(3): 74.

6. Azouz S, Walpole J, Amirifeli S, Taylor KN, Grinstaff MW, et al. (2008) Prevention of local tumor growth with paclitaxel-loaded microspheres. J Thorac Cardiovasc Surg 135(5): 1014-1021.

7. Blumen S, Cheng K, Ramos-Nino M, Taatjes DJ, Weiss DJ, et al. (2007) unique uptake of acid-prepared mesoporous spheres by lung epithelial and mesothelioma cells. Am J Respir Cell Mol Biol 36(3): 333-342.
8. Chao P, Deshmukh M, Kutscher H, Gao D, Rajan SS, et al. (2010) Pulmonary targeting microparticulate camptothecin delivery system: anti-cancer evaluation in a rat orthotopic lung cancer model. Anticancer Drugs 21(1): 65-76.

9. Sivadas N, Cryan S (2011) Inhalable, bioresponsive microparticles for targeted drug delivery in the lungs. J Pharm Pharmacol 63(3): 369-375.

10. Wang H, Xu Y, Zhou X (2014) Docetaxel-loaded chitosan microspheres as a lung targeted drug delivery system: in vitro and in vivo evaluation. Int J Mol Sci 15(3): 3519-3532.

11. Harsha S, Al-Dhubiab B, Nair A, Attimarad M, Venugopala MK, et al (2017) Pharmacokinetics and tissue distribution of microspheres prepared by spray drying technique: targeted drug delivery. Biomed Res 28(8): 3387-3396.

12. Harsha S, Al-Dhubiab B, Nair A, Al-khas M, Al-Hussain M, et al. (2015) Novel drying technology of microsphere and its evaluation for targeted drug delivery for lungs. Drying Technology 33(4): 502-512.

13. Fournier E, Passirani C, Colin N, Breton P, Sagodira S, et al. (2004) Development of novel 5-FU-loaded poly(methylidene malonate 2.1.2)-based microspheres for the treatment of brain cancers. Eur J Pharm Biopharm 57(2): 189-197.

14. Nagai T, Nishimoto Y, Nambu N, Suzuki Y, Sekine K (1984) Powder dosage form of insulin for nasal administration. J Control Release 1(1): 15-22.

15. Manjunatha N, Vasanti S, Rajesh N, Uma N (2010) Formulation and evaluation of biopolymer based microspheres for nasal drug delivery. Int J PharmTech Res 2(1): 856-862.

16. Pareta R, Edirisinghe M (2006) A novel method for the preparation of biodegradable microspheres for protein drug delivery. J R Soc Interface 3(9): 573-582.

17. Björk E, Erdman P (1990) Characterization of degradable starch microspheres as a nasal delivery system for drugs. Int J Pharm 62(23): 187-192.

18. Sun Y, Shi K, Wan F, deCui F (2012) Methotrexate-loaded microspheres for nose to brain delivery: in vitro/in vivo evaluation. J Drug Deliv Sci Technol 22(2): 167-174.

19. Shaji J, Poddar A, Iyer S (2009) Brain-targeted nasal clonazepam microspheres. Ind J Pharm Sci 71(6): 715-718.

20. Constantin M, Fundueanu G, Bortolotti F, Cortesi R, Ascenzi P, et al (2004) Preparation and characterization of poly(vinyl alcohol)/ cyclodextrin microspheres as matrix for inclusion and separation of drugs. Int J Pharm 285(1-2): 87-96.

21. Vallee J, Lo D, Guillevin R, Reb P, Adem C, et al. (2003) In vitro study of the compatibility of tris-acryl gelatin microspheres with various chemotherapeutic agents. J Vasc Interv Radiol 14(5): 621-628.

22. Laurent A (2007) Microspheres and nonspherical particles for embolization. Tech Vasc Interventional Rad 10(4): 248-256.

23. Liapi E, Kwang-Hun L, Georgiades C, Hong K, Geschwind J (2007) Drug-eluting particles for interventional pharmacology. Tech Vasc Interventional Rad 10(4): 261-269.

24. Kirchhoff T, Bleck J, Dettmer A, Chavan A, Rosenthal H, et al. (2007) Transarterial chemoembolization using degradable starch microspheres and iodized oil in the treatment of advanced hepatocellular carcinoma: evaluation of tumor response, toxicity, and survival. Hepatobiliary Pancreat Dis Int 6(3): 259-266.

25. Nguyen T, Tey S, Pourgholami M, David LM, Thomas PD, et al. (2007) Synthesis of semi-biodegradable crosslinked microspheres for the delivery of 1,25 dihydroxyvitamin D3 for the treatment of hepatocellular carcinoma. Eur Polym J 43(5): 1754-1767.

26. Yan Li \& Möhwald Templating Assembly of Multifunctional hybrid Colloidal Spheres. Advanced Materials 24(20): 2663-2667. 
CC (P) This work is licensed under Creative

(c) DY $10.19080 /$ GJPPS.2018.05.555664
Your next submission with Juniper Publishers will reach you the below assets

- Quality Editorial service

- Swift Peer Review

- Reprints availability

- E-prints Service

- Manuscript Podcast for convenient understanding

- Global attainment for your research

- Manuscript accessibility in different formats

( Pdf, E-pub, Full Text, Audio)

- Unceasing customer service

Track the below URL for one-step submission https://juniperpublishers.com/online-submission.php 